\title{
The effect of the second phase inversion on microstructures in phase inversion EVAL membranes
}

\author{
Tai-Horng Young, ${ }^{\mathrm{a}, *}$, Da-Ming Wang ${ }^{\mathrm{b}}$, Chih-Chen Hsieh ${ }^{\mathrm{b}}$, Leo-Wang Chen ${ }^{\mathrm{b}}$ \\ ${ }^{a}$ Center for Biomedical Engineering, College of Medicine, National Taiwan University, Taipei, Taiwan \\ ${ }^{\mathrm{b}}$ Department of Chemical Engineering, National Taiwan University, Taipei, Taiwan
}

Received 27 August 1997; received in revised form 17 March 1998; accepted 17 March 1998

\begin{abstract}
There were many discussions in the literature describing the membrane formation mechanism for the phase inversion process such as liquid-liquid demixing or crystallization, but few references described the phenomena after the event of the phase inversion process. The purpose of this work is to illustrate the effect of the second phase inversion on membrane structures when the first phase inversion has occurred. Analysis showed the second phase inversion (crystallization or liquidliquid demixing) may be preceded by the first phase inversion (liquid-liquid demixing only) during poly (ethylene-co-vinyl alcohol) (EVAL) membrane formation. Therefore, we can make membranes combined with macrovoids (the first phase inversion) and particulate morphology (the second phase inversion) from experiments in this work. As a result, the concept the membrane morphology only influenced by the liquid-liquid demixing is misleading and the second phase inversion must be considered as a possible and important mechanism. (C) 1998 Elsevier Science B.V. All rights reserved.
\end{abstract}

Keywords: Liquid-liquid demixing; Crystallization; The second phase inversion; Particulate morphology

\section{Introduction}

Nonsolvent-induced phase inversion process has been a standard technique to prepare polymeric membranes from an initially homogeneous polymer solution. During the process, the exchange of solvent and nonsolvent in the demixed film continues to cause an increase of the polymer concentration in the concentrated phase surrounding the pores. The polymer molecules may rearrange the wall structure when the vitrification of the concentrated phase does not

*Corresponding author. Tel.: 8862 23970800x1455; fax: 8862 23940049; e-mail: thyoung@ha.mc.ntu.edu.tw impede the wall growth [1]. Therefore, other phase separation types than liquid-liquid demixing such as crystallization may occur in the film [2], defined hereafter as the second phase inversion. The membrane structure is fixed until polymer molecules are unable to overcome the mechanical strength of the vitrified wall. Hence, the second phase inversion may reorganize the membrane morphology, which in turn governs the transport characteristics of the final membranes.

Several investigators have described the diffusion of solvent and nonsolvent in membrane solutions by a mass transport model [3-6]. According the model of Reuvers et al. [5], two types of demixing processes can be distinguished. If the composition profile in the 
membrane solution immediately crosses the binodal of the nonsolvent-solvent-polymer system after immersion of the solution into a nonsolvent bath, liquid-liquid demixing takes place immediately. On the other hand, if it takes a certain time for the composition profile to cross the binodal, the onset of liquid-liquid demixing is delayed. Unfortunately, a mass transport model only predicts the time at which a concentration path first crosses the binodal curve in the computation and all compositions existing in the polymer solution at the precipitation time. The prediction of the composition changes and its relation to the ultimate membrane structure after liquid-liquid demixing is beyond the scope of a mass transport model. Therefore, to understand the effect of the second phase inversion on the structure changes, detailed studies of the membrane formation were investigated in this paper. Significant results were indicated by changes in the phase inversion process under the specified conditions.

\section{Experiments}

The membrane material studied in this work is poly(ethylene-co-vinyl alcohol) (EVAL) (105A, Kuraray Japan) which contains ca. 56 mol\% vinyl alcohol [7]. Dimethyl sulfoxide (DMSO), isopropanol, and 1-octanol of extra pure reagent grade (Nacalai Tesque, Kyoto, Japan) were used as received. Water was double distilled and de-ionized before used.

Membranes were prepared using the direct immersion-precipitation method. An appropriate amount of EVAL was dissolved in DMSO to form a $25 \mathrm{wt} \%$ homogeneous solution. This solution was dispersed uniformly on a glass plate (ca. $100 \mu \mathrm{m}$ ) by an autocoater (KCC303, RK Print-Coat Instruments, UK), then precipitated in the nonsolvent bath to form a membrane. Freeze-dried samples of the membranes were examined using a scanning electron microscope (SEM) to obtain the membrane structures. In this study, specific nonsolvents were selected for evaluation of the effect of the second phase inversion on microstructures in phase inversion membranes. The nonsolvents include water, $20 \mathrm{wt} \%$ of DMSO and $80 \mathrm{wt} \%$ of water, isopropanol, $75 \%$ isopropanol and $25 \%$ water, and 1-octanol.

\section{Results and discussion}

\subsection{Membrane formation by liquid-liquid demixing}

In Fig. 1(a), the cross-section of the membrane formed by immersing a $25 \mathrm{wt} \%$ EVAL solution into a water bath is shown. This cross-section shows an asymmetrical structure consisting of the typical fingerlike macrovoids enclosed in a continuous polymer matrix, i.e., the liquid-liquid demixing dominates the membrane structure. The magnified cross-sectional view given in Fig. 1(b) indicates a cellular morphology between the macrovoids and the wall structure of macrovoids is very dense resembling the skin layer on the top surface of membranes. With the same procedure, but the composition of the coagulation bath is $20 \mathrm{wt} \%$ of DMSO and $80 \mathrm{wt} \%$ of water, the dense structure in the wall of macrovoids disappears (Fig. 2). On the contrary, the membrane has gradually porous structures from the top region to the bottom region in the wall of macrovoids. The difference in the wall structure of macrovoids can be ascribed to the fact that adding solvent into the coagulation bath can delay the phase separation and decrease the polymer concentration of the concentrated phase $[5,8]$. Therefore, it is favorable to proceed the second phase inversion for membrane solutions precipitated at a solvent-water bath. Conversely, it is to be expected that a highly concentrated solution grows out at the onset of liquid-liquid demixing for membrane prepared by a water bath to constrain the motion of polymer molecules and to fix immediately this asymmetric polymer distribution in the wall of macrovoids. In Fig. 1(b), no pores could be detected in the macrovoid wall at a magnification of $20000 \times$, indicating that no other phase inversion follows the first phase inversion. Thus, the polymer distribution that results from the diffusion processes at the onset of liquid-liquid demixing determines whether the second phase inversion proceeds [9].

Based on the above discussion, the resulting wall structure can be related to the second phase inversion. Once two separated phases are formed by liquidliquid demixing due to nucleation of the polymerpoor phase, the polymer-poor phase looks like to serve as a tiny coagulation bath [10] for the surrounding polymer-rich phase to proceed the second phase inversion. If the polymer-poor phase is like a water bath and 

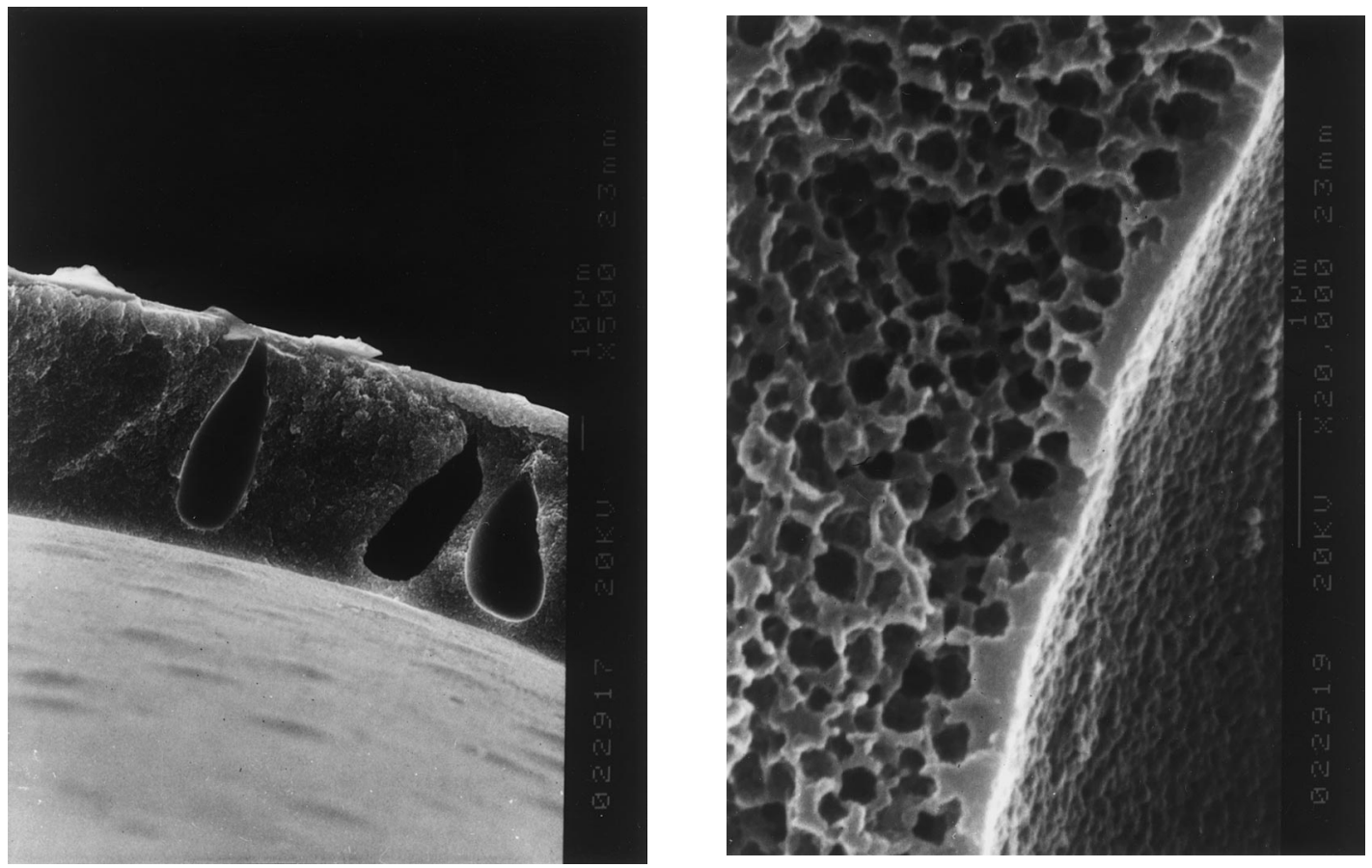

Fig. 1. (a) SEM photograph of the cross-section of the EVAL membrane immersed in a water bath. (b) The magnified photograph of (a) in the boundary of the macrovoid.

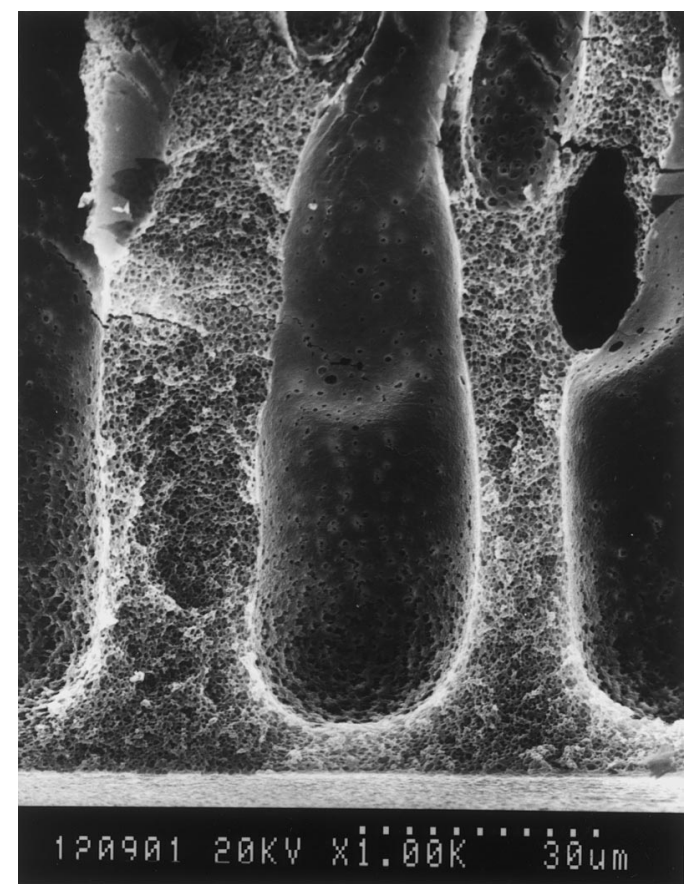

Fig. 2. SEM photograph of the macrovoid wall of the EVAL membrane immersed in a bath of $20 \%$ DMSO and $80 \%$ water. the polymer-rich phase is formed by the rapid vitrification, then the wall of pores will be rapidly fixed to form a dense structure and the second phase inversion has no chance to occur. On the other hand, if the polymer-poor phase is like a solvent-water bath and the wall is formed by the mechanism of delayed onset of vitrification, then the second phase inversion is likely to happen. Therefore, a microporous wall will be formed when the new nuclei are formed (can be considered as the second phase inversion) just close to the wall, which can grow through the wall. Obviously, addition of solvent to the coagulation bath is able to account for the difference in these two cases.

\subsection{Combination of crystallization and liquid-liquid demixing}

In contrast to the cellular morphology mentioned above for EVAL membranes coagulated in a water bath, membranes are characterized by the so-called "particulate" morphology when EVAL solutions are immersed into an isopropanol bath. The overall crosssection of the formed membrane is shown in Fig. 3(a) 

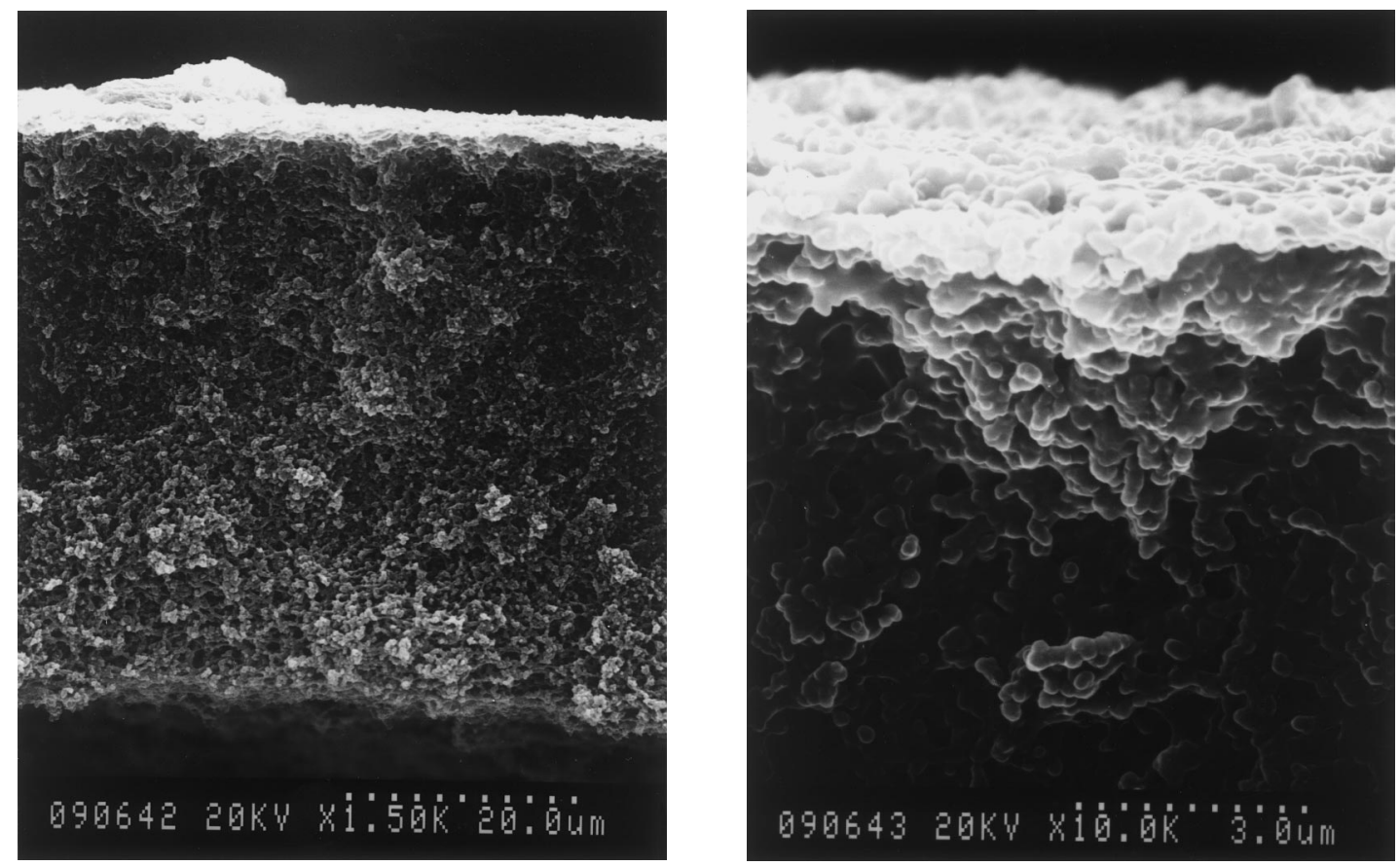

Fig. 3. (a) SEM photograph of the cross-section of the EVAL membrane immersed in an isopropanol bath. (b) The magnified photograph of (a) near the top layer.

and a magnified view is given in Fig. 3(b). The distribution of particles is uniform throughout the entire membrane. This suggests that all of the particles are emerged from individual EVAL nuclei born roughly simultaneously. These nuclei then grow radially until their fronts attach and join with adjacent particles to form a skinless and microporous structure. In Fig. 3, there is no evidence of the cellular morphology that is initiated by the nucleation of liquid-liquid demixing. Therefore, the formation of particles is the dominant mechanism to form a microporous membrane. With different circumstances, there can be three possible mechanisms for the formation of particles in membranes. The first possibility is that particles are generated by the nucleation and growth of the polymerrich phase resulted from liquid-liquid demixing when the polymer concentration is lower than the critical point of binodal $[11,12]$. The second possible origin of particles is spinodal demixing [13]. The third possibility comes from the consideration that crystallization of polymer during the membrane formation process is responsible for the formation of particles
$[2,7,14,15]$. In general, the location of the critical point is at very low polymer concentration. Hence the presence of particles by the nucleation and growth of the polymer-rich phase is only at a dilute polymer solution. In addition, the building units of EVAL membranes are particles of approximately equal size (ca. $0.3 \mu \mathrm{m}$ dia.) which is greater than the size of nodules caused by the spinodal demixing. Thus, at present, it must be suggested that EVAL membranes with unexpected particles-bonded together morphology is dominated by a crystallization-induced process [7].

In fact, EVAL is a crystalline polymer that can be isothermally precipitated from a solution either by a liquid-liquid demixing or by a crystallization process [7]. This type of nonsolvent is an important factor that determines which precipitation sequence is kinetically favored in the immersion-precipitation process. Fig. 4 shows the morphology of membranes, coagulated in a bath of $75 \%$ isopropanol and $25 \%$ water, is a combination of macrovoids and particles with the size about $0.5 \mu \mathrm{m}$. From the membrane structure, the phase 

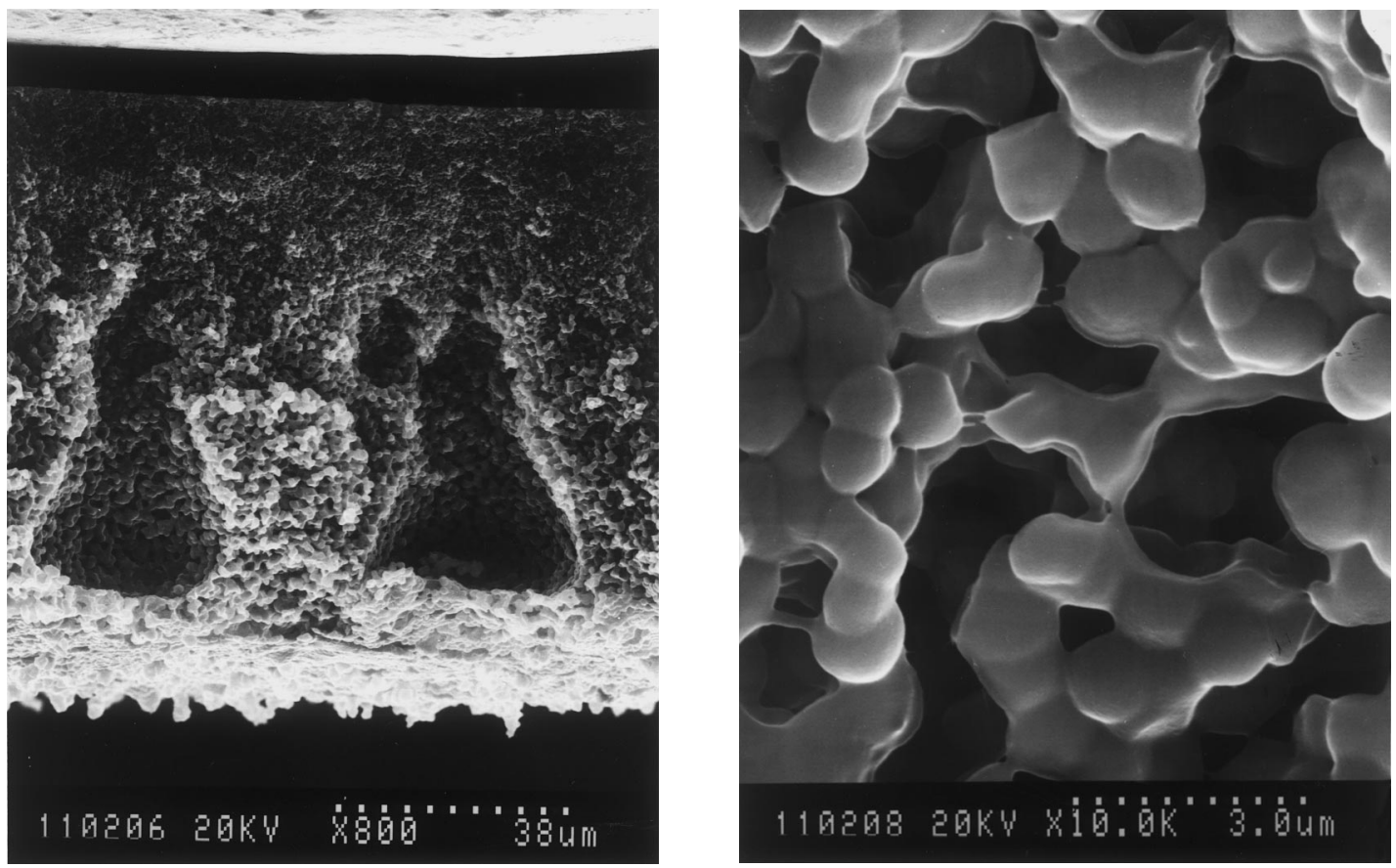

Fig. 4. (a) SEM photograph of the cross-section of the EVAL membrane immersed in a bath of $25 \%$ water and $75 \%$ isopropanol. (b) The magnified photograph of (a) in the macrovoid wall.

separation for this membrane may be either due to the onset of liquid-liquid demixing or crystallization. If crystallization takes place prior to liquid-liquid demixing during the membrane formation, as described previously, EVAL molecules will form particles distributing in the membrane. However, it is impossible for these particles are clustered together to reorganize their distribution from within their domains to form macrovoids, i.e., particles will be fixed in their location to suppress the tendency of liquid-liquid demixing. This suggests that the phase separation is initiated by liquid-liquid demixing. Following this process, the polymer-rich phase in the demixed film remains in a metastable state with respect to crystallization [7]. Prior to the vitrification of the concentrated phase, EVAL molecules distribute themselves to form crystallization-induced particles, which consist of a number of macromolecules entangled and overlapping each other. When these particles come close together they form void spaces among them, the voids will give rise to larger pores and the empty spaces within each particle will give rise to small pores [16].
Consequently, the total membrane structure is composed of macrovoids originated from liquid-liquid demixing and the wall structure surrounding the pores are particulate morphology. Although the liquidliquid demixing dominates the overall membrane structure, the crystallization (the second phase inversion) changes the resultant morphology from an asymmetric to a microporous membrane.

Overall, for semicrystalline polymers, both liquidliquid demixing and crystallization may occur during the membrane formation [17]. From a kinetic consideration, the crystallization process which need a longer induction period will not be operative [10]. Consequently, it is easily imagined that the membrane morphology is determined by liquid-liquid demixing. We believe that it is not reasonable to neglect the effect of the crystallization process on the membrane morphology, especially, crystallization of polymers might occur in the second step of membrane formation due to the polymer-rich phase generally with a large supersaturation. We do agree with Cheng et al. [2], who proposed that crystallization process even might occur 

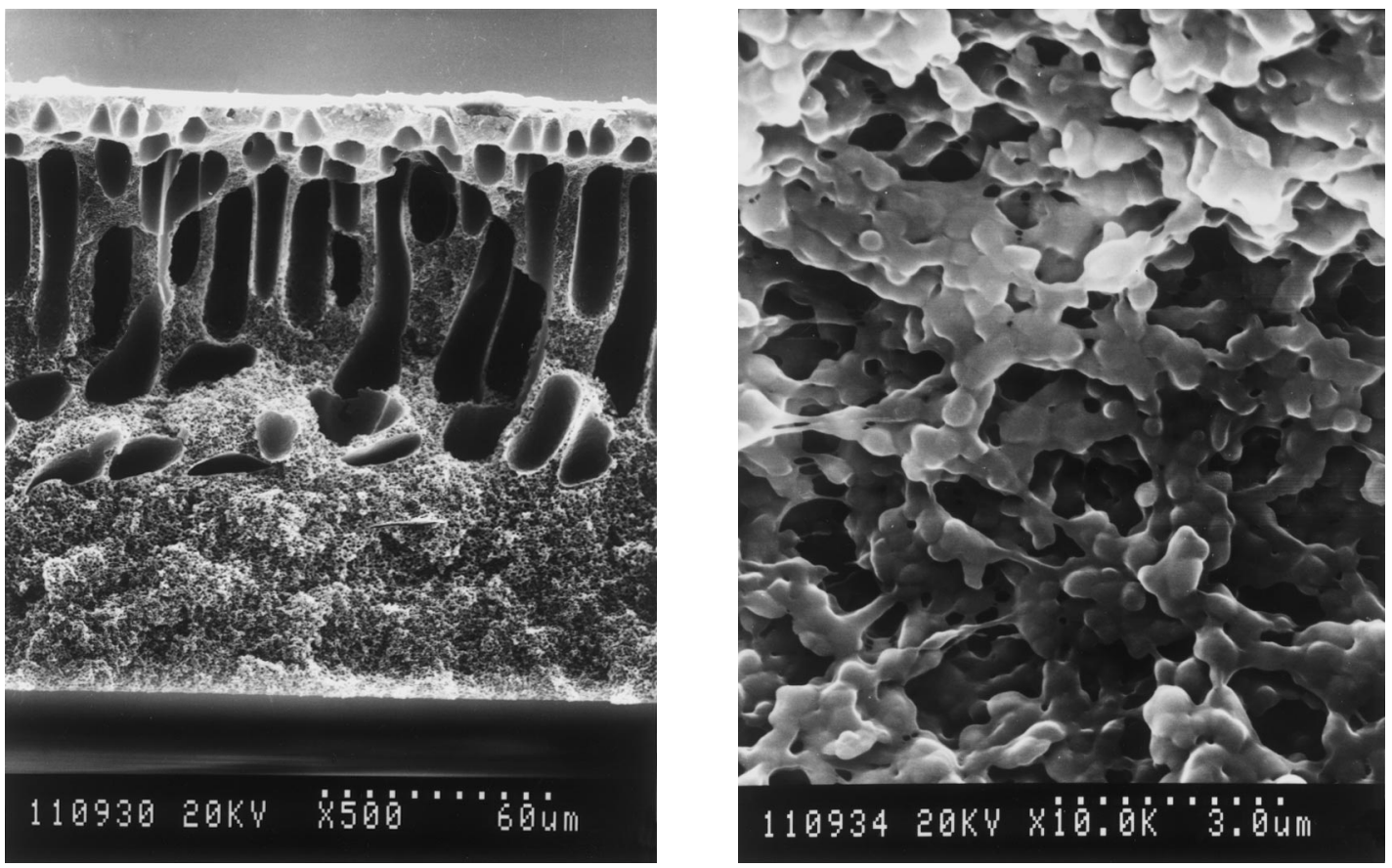

Fig. 5. (a) SEM photograph of the cross-section of the EVAL membrane prepared by immersion into a $20 \%$ DMSO/80\% water bath for $5 \mathrm{~s}$ followed by a 1-octanol bath. (b) The magnified photograph of (a) in the macrovoid wall.

before liquid-liquid demixing. Therefore, the second phase inversion might form the particle morphology in the wall, thereby modifying nucleus wall to change the transport characteristics of the membranes.

\subsection{Membrane formation by a dual-bath method}

The dual-bath method was employed to control the membrane structure and to investigate whether or not the first phase inversion can give enough rigidity to form a solid membrane structure. The first bath was a solution of $80 \%$ water and $20 \%$ DMSO and the second bath was 1-octanol only. The polymer solution was immersed in the first bath immediately after casting and kept there for a predetermined period, permitting the first phase inversion to occur. Then the membrane solution was placed into the second bath for more than $12 \mathrm{~h}$, where the second phase inversion proceeded to fulfill the overall membrane.

When the casting solution is immersed in the first bath for $5 \mathrm{~s}$ followed by the second bath, the top region of the cross-section shows evidence of macrovoids due to the liquid-liquid demixing (Fig. 5(a)). The wall structure of macrovoids has an obvious particulate morphology (Fig. 5(b)). The nonsolvent of the second bath, 1-octanol, like isopropanol, can produce EVAL membranes with particulate morphology [16]. Therefore, the polymer-rich phase surrounding the macrovoids prepared in the first bath becomes supersaturated with respect to the crystallization equilibrium in the second bath. Consequently, the wall structure of macrovoids are changed by 1-octanol from a porous morphology (Fig. 2) dominated by the liquid-liquid demixing to a crystallization-induced particulate morphology. On the contrary, if the casting solution is immersed in a 1-octanol bath for $5 \mathrm{~s}$ followed by a $20 \%$ DMSO bath, the membrane is skinless and uniform composed of equal-sized spherical particles (Fig. 6) which resembles the membrane coagulated in 1-octanol only (see Fig. 1(e) in Ref. [16]). This shows when the particle nuclei is formed in the first 1-octanol bath, there is no chance for polymer molecules to proceed liquid-liquid demixing as the second phase inversion to form macrovoids in the membrane.

In addition, the dual-bath method can be used to control the growth of macrovoids. As the polymer 

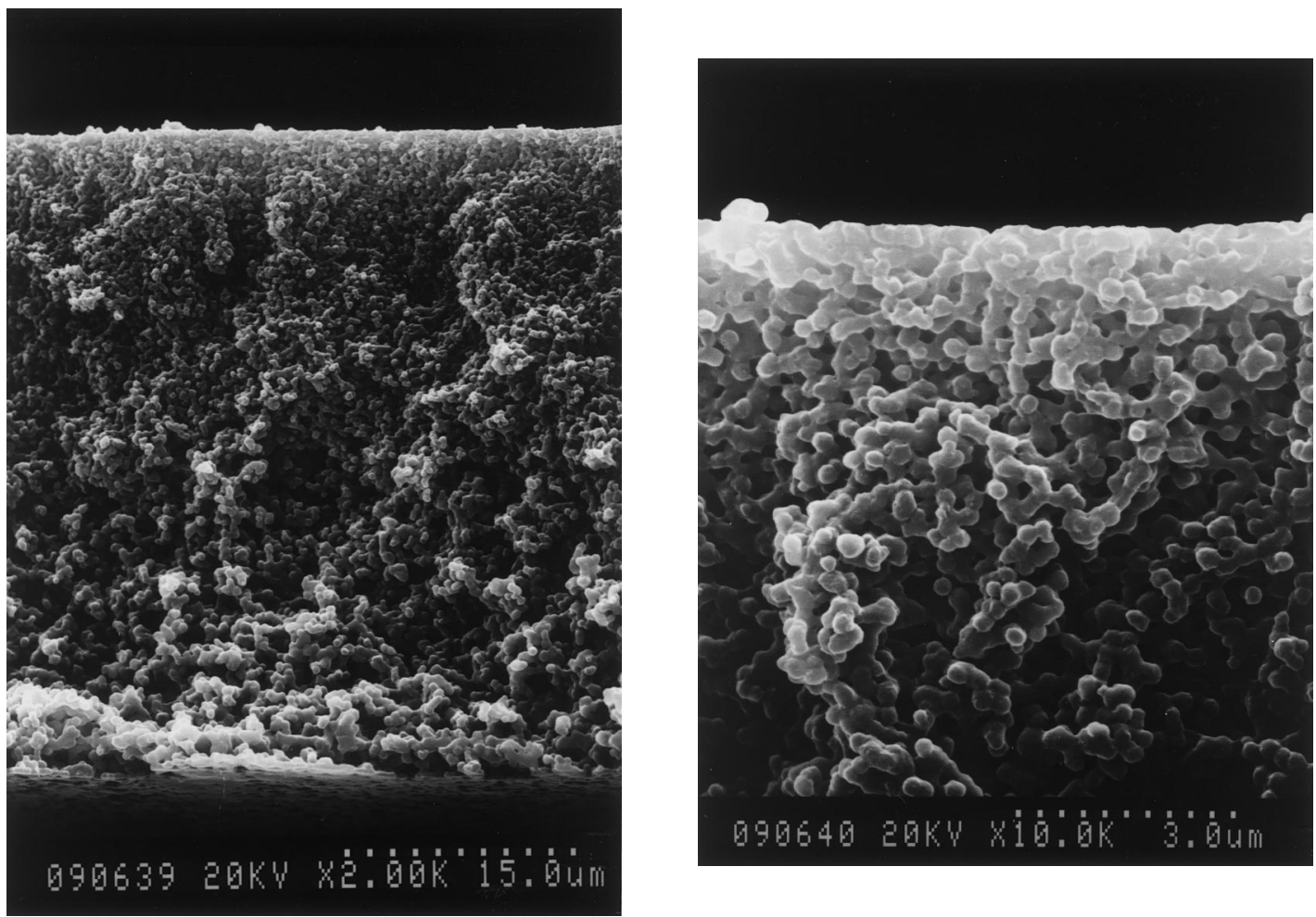

Fig. 6. (a) SEM photograph of the cross-section of the EVAL membrane prepared by immersion into a 1-octanol bath for $5 \mathrm{~s}$ followed by a $20 \%$ DMSO/80\% water bath. (b) The magnified photograph of (a) near the top layer.

solution contacts nonsolvent of the first bath, the liquid-liquid demixing starts to take place and the macrovoids initiate and grow inward. However, macrovoids which grow in the first bath will stop growing in the 1-octanol bath. Hence, by regulating the time of the membrane solution in the first bath, we can control the degree of the first phase inversion, i.e., the size of macrovoids. Fig. 7 shows the macrovoids grow continuously with an increase in the coagulation time of the first bath. If the system is assumed to follow a semi-infinite slab transport kinetics until the bottom plate is encountered by the growth of macrovoids, then the length of macrovoids can be plotted against the square root of time in the first bath to investigate the growth mechanism of macrovoids. Fig. 8 shows that a linear relationship is found. It indicates that the growing macrovoid can be considered as a result of diffusional flow rather than of convective flows [18]. From the slope of line in Fig. 8, the apparent diffusivity for the macrovoid growth could be calculated by the equation suggested by Strathmann et al. [19] and Kang et al. [20], resulting in $2.8 \times 10^{-10} \mathrm{~m}^{2} / \mathrm{s}$. This is in accordance with the results of other authors using the optical microscope to measure the growth of macrovoids [19-21]. In other words, for the growth of macrovoids, the liquid-liquid demixing is instantaneous only beneath the top layer, while the polymer concentration may hardly change in the region away from the interface of the membrane solution. Therefore, the formation of macrovoids is a delayed onset of demixing at a certain distance from the film interface and other phase inversions are expected to interfere the growth of macrovoids. Consequently, the particulate morphology will have the opportunity to replace the cellular morphology in the wall of macrovoids, between macrovoids and below macrovoids. Such a result implies that the macrovoids grow by a diffusional flow that results from the first phase inversion (liquid-liquid demixing) and the wall of 

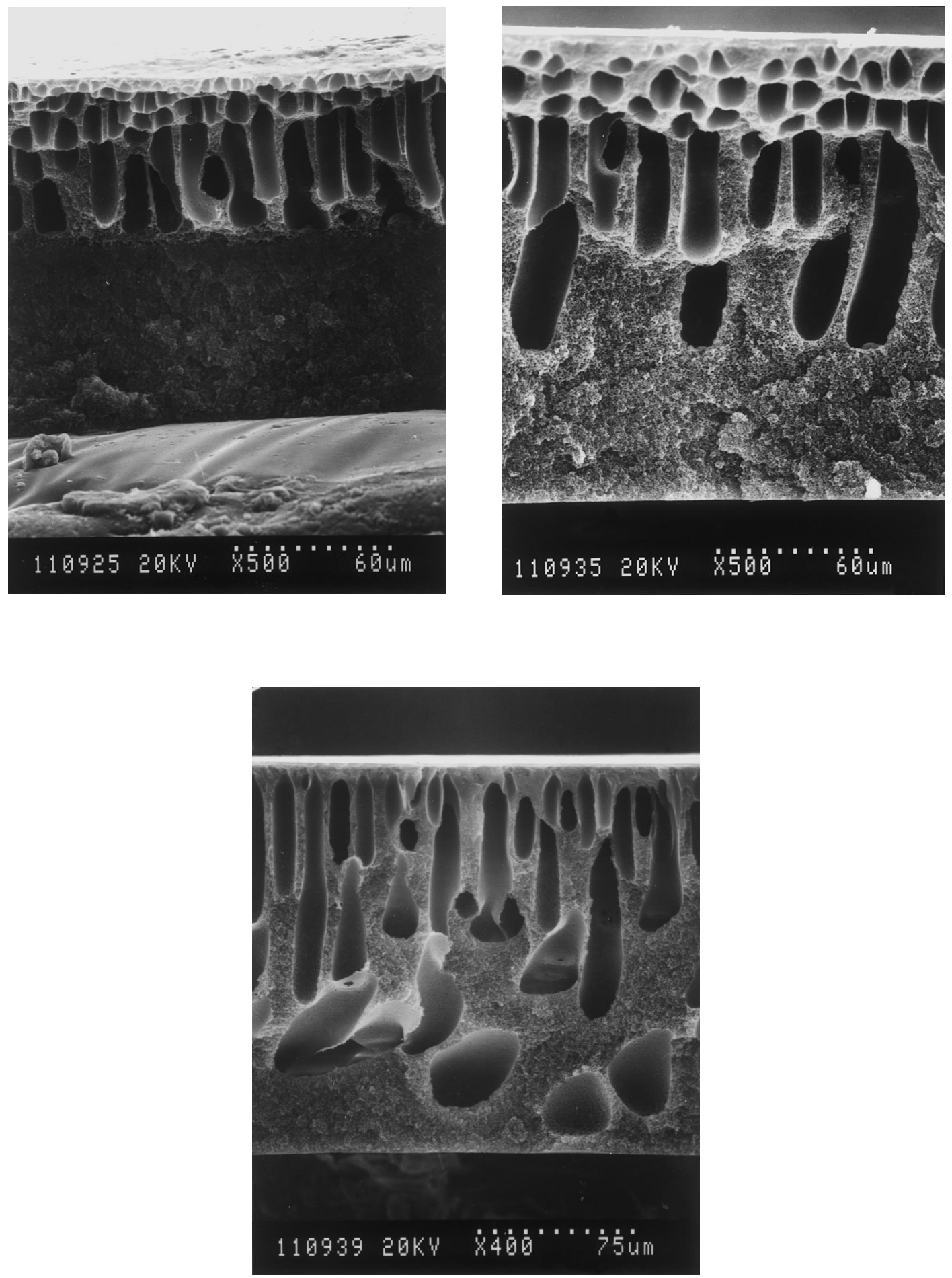

Fig. 7. SEM photographs of the cross-section of the EVAL membrane prepared by immersion into a $20 \%$ DMSO/80\% water bath for different times followed by a 1-octanol bath. (a) $1 \mathrm{~s}$, (b) $10 \mathrm{~s}$ and (c) $20 \mathrm{~s}$. (The SEM photograph for $5 \mathrm{~s}$ is shown in Fig. 5.) 


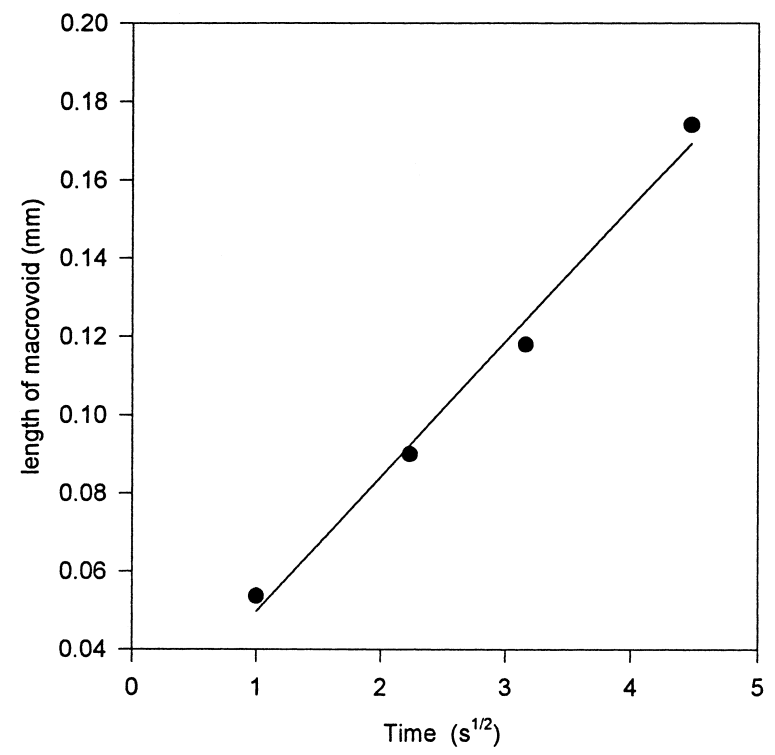

Fig. 8. A plot of the longitudinal length of macrovoid vs. square root of time in the $20 \%$ DMSO/80\% water bath, followed by a 1 octanol bath.

macrovoids is from the second phase inversion (crystallization).

\section{Conclusions}

The purpose of this work is to illustrate that the second phase inversion have the opportunity to occur after the first phase inversion. If the first phase inversion is due to crystallization, further liquid-liquid demixing is prevented. Conversely, if the first phase inversion is due to liquid-liquid demixing, the second phase inversion will probably happen. The second phase inversion can be dominated by crystallization or liquid-liquid demixing. As a result, the concept the membrane morphology only influenced by the liquidliquid demixing is misleading and the second phase inversion must be considered as a possible and important mechanism.

\section{Acknowledgements}

The authors thank the National Science Council of the Republic of China for their financial support of project NSC 86-2216-E-002-003.

\section{References}

[1] A.J. Reuvers, C.A. Smolders, Formation of membranes by means of immersion precipitation, Part II. The mechanism of formation of membranes prepared from the system celluloseacetone-water, J. Membr. Sci. 34 (1987) 67-86.

[2] L.P. Cheng, A.W. Dwan, C.C. Gryte, Membrane formation by isothermal precipitation in polyamide-formic acid-water systems description of membrane morphology, J. Polym. Sci. Polym. Phys. 33 (1995) 211-222.

[3] C. Cohen, G.B. Tanny, S.J. Prager, Diffusional-controlled formation of porous structures in ternary polymer systems, J. Polym. Sci., Part A-2 17 (1979) 477-489.

[4] L. Yilmaz, A.J. McHugh, Modeling of asymmetric membrane formation. I. Critique of evaporation models and development of a diffusion equation formalism for the quench period, J. Membr. Sci. 28 (1986) 287-310.

[5] A.J. Reuvers, J.W.A. van der Berg, C.A. Smolders, Formation of membranes by means of immersion precipitation. Part I. A model to describe mass transfer during immersion precipitation, J. Membr. Sci. 34 (1987) 45-65.

[6] P. Radovanovic, S.W. Thiel, S.-T. Hwang, Formation of asymmetric polysulfone membranes by immersion precipitation. Part I. Modeling mass transport during gelation, J. Membr. Sci. 65 (1992) 213-229.

[7] T.H. Young, J.Y. Lai, W.M. Yu, L.P. Cheng, Equilibrium phase behavior of the membrane forming water-DMSOEVAL copolymer system, J. Membr. Sci. 128 (1997) 55-65.

[8] M. Mulder, Basic Principles of Membrane Technology, Kluwer Academic Publishers, Dordrecht, Netherlands, 1991.

[9] G.E. Gaides, A.J. McHugh, Gelation in an amorphous polymer: a discussion of its relation to membrane formation, Polymer 30 (1989) 2118-2123.

[10] C.A. Smolders, A.J. Reuvers, R.M. Boom, I.M. Wienk, Microstructures in phase inversion membranes. Part 1. Formation of macrovoids, J. Membr. Sci. 73 (1992) 259-275.

[11] T.H. Young, L.W. Chen, L.P. Cheng, Membranes with a microparticulate morphology, Polymer 37 (1996) 1305-1310.

[12] K. Kamide, S. Manabe, in: D.R. Lloyd. (Ed.), Material Science of Synthetic Membranes, American Chemical Society, Washington, DC, 1985, pp. 197-228.

[13] S.P. Nunes, T. Inoue, Evidence for spinodal decomposition and nucleation and growth mechanisms during membrane formation, J. Membr. Sci. 111 (1996) 93-103.

[14] A.M.W. Bulte, B. Folkers, M.H.V. Mulder, C.A. Smolders, Membranes of semicrystalline aliphatic polyamide nylon 4,6: formation by diffusion-induced phase separation, J. Appl. Polym. Sci. 50 (1993) 13-26.

[15] L.P. Cheng, A.W. Dwan, C.C. Gryte, Membrane formation by isothermal precipitation in polyamide-formic acid-water systems. II. Precipitation dynamics, J. Polym. Sci. Polym. Phys. 33 (1995) 223-235.

[16] L.P. Cheng, H.Y. Lin, L.W. Chen, T.H. Young, Solute rejection of dextran by EVAL membranes with asymmetric and particulate morphologies, Polymer 39 (1988) 2135-2142.

[17] A.M.W. Bulte, M.H.V. Mulder, C.A. Smolders, H. Strathmann, Diffusion induced phase separation with crystallizable 
nylons. I. Mass transfer processes for nylon 4,6, J. Membr. Sci. 121 (1996) 37-49.

[18] J. Crank, The Mathematics of Diffusion, Oxford University Press, London, 1975.

[19] H. Strathmann, K. Kock, P. Amar, R.W. Baker, The formation mechanism of asymmetric membranes, Desalination 16 (1975) 179-203.
[20] Y.S. Kang, H.J. Kim, U.Y. Kim, Asymmetric membrane formation via immersion precipitation method. I. Kinetic effect, J. Membr. Sci. 60 (1991) 219-232.

[21] H.J. Kim, R.K. Tyagi, A.E. Fouda, K. Jonasson, The kinetic study for asymmetric membrane formation via phase-inversion process, J. Appl. Polym. Sci. 62 (1996) 621-629. 